\title{
Field Dependence of Spin Lifetimes in Wurtzite Materials
}

\author{
J.A. MajewSKI* \\ Institute of Theoretical Physics, Warsaw University \\ 00-681 Warszawa, Hoża 69, Poland
}

\begin{abstract}
We present first-principles calculations of the zero field spin splitting of energy bands in wurtzite materials. Our calculations reveal that the huge electric fields originating from strong piezo- and pyroelecric character of nitrides do not increase the spin splitting of bands in nitride heterostructures. This implicates long spin lifetimes in quantum structures based on these materials and weak possibility of tuning with external electric field.
\end{abstract}

PACS numbers: 71.15.Mb, 71.20.Nr, 71.70.Ej, 71.70.Fk

\section{Introduction}

Recently, the room temperature ferromagnetism in diluted magnetic semiconductor GaN:Mn has been predicted theoretically and later confirmed in numerous experiments [1]. This opens new perspectives for spin devices entirely based on nitrides. The possible design of such spintronic devices requires the understanding of the spin dynamics and spin relaxation processes, which are, in turn, determined by the zero field spin splitting of the bands. Unfortunately, the spin splitting of the bands, induced by the spin orbit interaction, is mostly unknown in nitride quantum structures. In standard semiconductors, the electric field dependence of the spin splitting of energy bands (Rashba effect) has a marked influence on the spin dynamics and spin relaxation processes. This issue becomes much more relevant in nitride heterostructures. In view of their huge (of the order of a few $\mathrm{MV} / \mathrm{cm}$ ) electric fields that originate in their strong piezo- and pyroelectric character, one may anticipate a very significant spin splitting.

In this paper, we present a systematic study of the influence of these built-in electric fields on the spin splitting in nitride heterostructures. These studies are based on first-principles, relativistic local density calculations that provide very accurate description of the spin splitting in nitride bulks $[2,3]$. Here, we concentrate on the lowest order in the wave vector $\boldsymbol{k}$ spin splitting, i.e., terms linear in $\boldsymbol{k}$.

*e-mail: Jacek.Majewski@fuw.edu.pl 


\section{Results and discussion}

\subsection{Zero field spin splitting in wurtzite bulks}

For wurtzite bulks and superlattices grown along the $c$-axis, the effective $2 \times 2$ Hamiltonian describing linear- $k$ terms can be written in the form $H=\boldsymbol{\sigma} \cdot \boldsymbol{B}_{\text {eff }}$, with $\boldsymbol{B}_{\text {eff }}\left(k_{x}, k_{y}\right)=\alpha_{R}\left(k_{y},-k_{x}, 0\right)$, where $\boldsymbol{\sigma}$ is the vector of Pauli matrices, $k_{x}$ and $k_{y}$ are wave vector components in the plane perpendicular to the hexagonal axis, and $\alpha_{R}$ is a band dependent constant that determines the isotropic spin splitting energy $\Delta E_{\text {spin }}=2 \alpha_{R}\left(k_{x}^{2}+k_{y}^{2}\right)^{1 / 2}$ of a certain band. In the wurtzite structure, the combination of crystal-field and spin-orbit interactions leads to a three-edge structure of the top of the valence band in the $\Gamma$ point, known as A, B, and C edges. Two of these three edges are of $\Gamma_{7}$ and one of $\Gamma_{9}$ symmetry, while the lowest

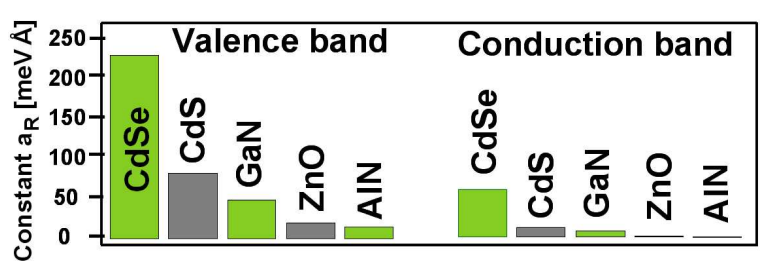

Fig. 1. Linear- $k$ spin splitting (in $\mathrm{eV} \AA$ ) of the conduction band and the higher $\Gamma_{7}$ valence band in a few wurtzite semiconductors.

conduction state has $\Gamma_{7}$ symmetry. The valence states are, in order of decreasing energy, $\Gamma_{9}, \Gamma_{7}, \Gamma_{7}$ for GaN, and $\Gamma_{7}, \Gamma_{9}, \Gamma_{7}$ for AlN (i.e., like in $\mathrm{ZnO}$ ). It is known that the $\Gamma_{9}$ states of wurtzite are not linearly split. In Fig. 1 we depict calculated values of constant $\alpha_{R}$ for the highest $\Gamma_{7}$ valence and conduction band states in AlN and GaN and compare with the values for other semiconductors. As can be seen in Fig. 1, in all wurtzite semiconductors, the linear- $k$ spin splitting is considerably smaller in the conduction band than in the valence band.

\subsection{Zero filed linear-k spin splitting in AlN/GaN superlattices}

In further discussion, we focus on the spin splitting of the conduction band in AlN/GaN superlattices. We have performed calculations for AlN $/(\mathrm{GaN})_{n}$ superlattices with various widths of the GaN quantum wells, measured by the number of GaN layers $n$. The divergence of the large electric polarization (spontaneous and piezoelectric) causes negative and positive bound sheet charges located at the interfaces, which leads to huge electric fields in the structure of the order of a few $\mathrm{MV} / \mathrm{cm}$. A schematic band line-up of $(\mathrm{AlN})_{4} /(\mathrm{GaN})_{8}$ superlattice together with the calculated wave functions of the top of the valence band and bottom of the conduction band are shown in Fig. 2. The wave function of the valence band top is strongly localized at one interface, just giving rise to the negative polarization charge. In the $(\mathrm{AlN})_{4} /(\mathrm{GaN})_{n}$ superlattices on GaN substrate, the calculated values of the constant $\alpha_{R}$ are equal to $0.4,0.9,0.6$, and $0.8 \mathrm{meV} \AA$ for $n=4,8,12$, 


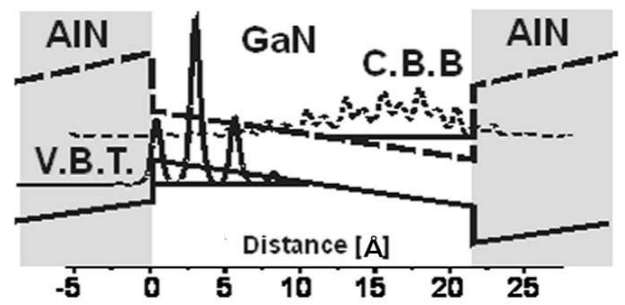

Fig. 2. Band line-up and wave functions of the valence (VBT) and conduction band (CBB) edges. For both bands, the laterally averaged first-principles wave-functions are shown.

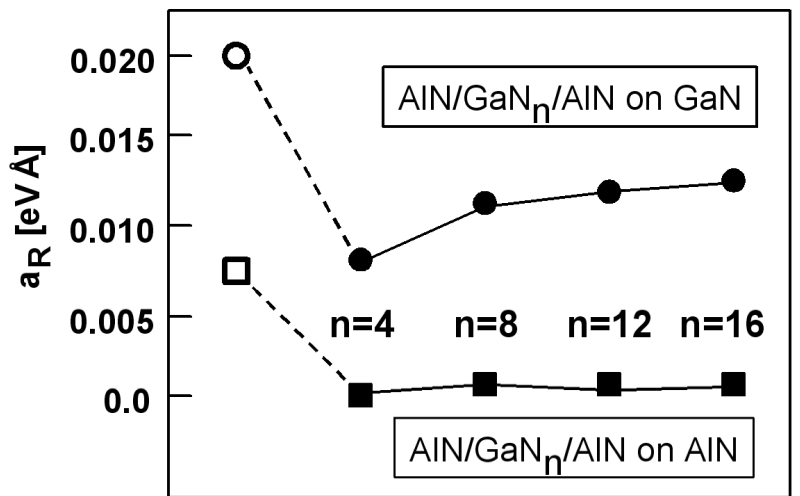

Fig. 3. Linear- $k$ spin splitting of the conduction band (in eV $\AA$ ) in $\mathrm{AlN}_{4} / \mathrm{GaN}_{n}$ superlattices for various thickness of the quantum well measured in a number of GaN double layers $n$. Full circles indicate superlattices with strained quantum well, i.e., grown on AlN, whereas full squares indicate superlattices with unstrained quantum wells, i.e., grown on GaN. For comparison, the values of the $\alpha_{R}$ constants for unstrained GaN bulk (square) and biaxially strained GaN (circle) are also given. The lines are drawn to guide the eye.

16, respectively (see Fig. 3). They are therefore an order of magnitude smaller than the corresponding value of the $\alpha_{R}$ constant for bulk GaN ( $\alpha_{R}=9 \mathrm{meV} \AA$ ). It means that the macroscopic electric field in the well that originates from interfaces charges counteracts the effect of spontaneous polarization of the bulk material and leads to the strong reduction of the conduction band spin splitting. This reduction is only weakly dependent on the width of the well.

To investigate the role of the macroscopic electric field further, we calculated also the spin splitting in the $\mathrm{AlN} /(\mathrm{GaN})_{n}$ superlattices grown on AlN. In such structures GaN quantum wells are subject to the strong compressive biaxial strain that results from the lattice mismatch $(-2.5 \%)$. This strain causes the considerable increase of the conduction band spin splitting, giving the value of $\alpha_{R}$ equal to $20 \mathrm{meV} \AA$ in biaxially strained GaN. For the $(\mathrm{AlN})_{4} /(\mathrm{GaN})_{n}$ superlattices on 
AlN, the calculations yield the values of $\alpha_{R}$ equal to 8,11 , and $12 \mathrm{meV} \AA$ for $n=4,8,16$, respectively. The reduction of $\alpha_{R}$ constant caused by the polarization induced macroscopic electric field is of the same order as in the case of AlN/GaN superlattices grown on GaN.

We would like to stress that the linear in $\mathrm{k}$ spin splitting terms dominate the spin splitting around the $\Gamma$ point. Even for wave vectors of the length $0.25 \AA^{-1}$ (roughly $1 / 4$ of the Brillouin zone) the linear term constitutes approximately $75 \%$ of the total spin splitting. The strong dependence of the constant $\alpha_{R}$ on the growth induced strain (mostly unknown) hinders direct comparison with experiment. On the other hand, the present calculations shed light on the physical origin of contradictory values of the spin splitting of the conduction band electrons as extracted from various experiments [4-6].

Altogether, the conduction band spin splitting of the AlN/GaN heterostructures remains small in spite of the huge electric fields, indicating long spin relaxation times in the nitride heterostructures. For comparison, $\alpha_{R}$ for [111] $\mathrm{AlAs} / \mathrm{GaAs}$ heterostructure is by the factor 10 larger (equal to $140 \mathrm{meV} \AA$ ) than the highest value of $\alpha_{R}$ observed in AlN/GaN structures. On the other hand, the spin splitting of the conduction band can be only marginally influenced by the external electric field. The calculations show that the external bias of $250 \mathrm{kV} / \mathrm{cm}$ changes the constant $\alpha_{R}$ by approximately $1 \%$.

\section{Conclusions}

The presented calculations strongly suggest that GaN heterostructures are a promising candidate for some spintronic applications, where long spin lifetime is needed. GaN quantum wells are not particularly suitable for spin transistor (rather weak steering possibility) but they can be very good for static Qbits.

\section{References}

[1] T. Dietl, H. Ohno, F. Matsukara, J. Cibert, D. Ferrand, Science 287, 139 (1998); for a review of experiments see T. Graf, T.B. Goennenwein, M.S. Brandt, Phys. Status Solidi B 239, 277 (2003).

[2] J.A. Majewski, M. Städele, P. Vogl, Mat. Res. Soc. Symp. Proc. 449, 887 (1997).

[3] J.A. Majewski, S. Birner, A. Trellakis, M. Sabathil, P. Vogl, Phys. Status Solidi C 1, 2003 (2004).

[4] K. Tsubaki, N. Maeda, T. Saitoh, N. Kobayashi, Appl. Phys. Lett. 80, 3126 (2002).

[5] B. Beschoten, E. Johnston-Halperin, D.K. Young, M. Poggio, J.E. Grimaldi, S. Keller, S.P. DenBaars, U.K. Mishra, E.L. Hu, D.D. Awschalom, Phys. Rev. B 63, 21202 (2001).

[6] C.K. Choi, J.B. Lam, G.H. Gainer, S.K. Shee, J.K. Krasinski, J.J. Song, Phys. Rev. B 65, 155206 (2002). 